\title{
Expression of glia maturation factor $\gamma$ is associated with colorectal cancer metastasis and its downregulation suppresses colorectal cancer cell migration and invasion in vitro
}

\author{
HUILI WANG ${ }^{1,2}$, ZHIJIANG CHEN $^{3}$, HONGEN CHANG $^{4}$, XIAOPING MU ${ }^{2}$, WENYU DENG $^{2}$, \\ ZHAOHU YUAN ${ }^{5}$, FANG YAO ${ }^{1}$, YAN LIU ${ }^{2}$, RONGJIA MAI ${ }^{2}$ and BINGYI WU ${ }^{1}$ \\ ${ }^{1}$ Research Center of Clinical Medicine, Nanfang Hospital, Southern Medical University, Guangzhou 510000; \\ ${ }^{2}$ Guangdong Women and Children Hospital, Guangzhou 510000; ${ }^{3}$ Pediatric Center of Zhujiang Hospital, \\ Southern Medical University, Guangzhou 510000; ${ }^{4}$ Department of Neurology, Liuzhou Hospital of \\ Traditional Chinese Medicine, Guangxi 545001; ${ }^{5}$ Department of Blood Transfusion Center, \\ Guangzhou First People's Hospital, Guangzhou Medical University, \\ Guangzhou 510000, P.R. China
}

Received June 20, 2016; Accepted November 11, 2016

DOI: $10.3892 / o r .2017 .5361$

\begin{abstract}
Glia maturation factor $\gamma$ (GMFG) functions to reorganize the actin cytoskeleton and appears to play a causative role in cell migration and adherence. The present study assessed GMFG expression in colorectal cancer cells and tissue specimens and then explored the role of GMFG in colorectal cancer progression in vitro. GMFG protein was highly expressed in colorectal cancer tissues and a metastatic colon cancer cell line. Knockdown of GMFG expression using GMFG siRNA or anti-GMFG antibody decreased the capacity of colon cancer LoVo cell migration and invasion in vitro, while recombinant GMFG treatment induced LoVo cell migration. Furthermore, GMFG knockdown also decreased expression of MMP2 protein and reversed epithelial-mesenchymal transition (EMT) phenotypes in LoVo cells. Co-culture of LoVo cells with human umbilical vein endothelial cells (HUVECs) and exogenous GMFG treatment promoted LoVo cell migration and invasion. The data from the present study indicate that GMFG should be further evaluated as a biomarker for detection of colorectal cancer metastasis and that the targeting
\end{abstract}

Correspondence to: Dr Bingyi Wu, Research Center of Clinical Medicine, Nanfang Hospital, Southern Medical University, 1838 Baiyun Avenue North, Guangzhou 510000, P.R. China E-mail: nfyywubingyi@163.com

Abbreviations: GMFG, glia maturation factor $\gamma$; MMP2, matrix metalloproteinase 2; EMT, epithelial-mesenchymal transition; E-Cad, E-cadherin; ADF, actin-depolymerizing factor

Key words: colorectal cancer, GMFG, MMP2, EMT, metastasis, invasion of GMFG expression or function could be a novel strategy in the future control of colorectal cancer.

\section{Introduction}

Colorectal cancer is the third most commonly occurring cancer worldwide and accounts for the third highest cancerrelated mortality rate in the US $(1,2)$. Colorectal cancer development may take decades by transformation of normal epithelial cells to invasive tumor cells and involves many genetic alterations and is influenced by many environmental factors. Dr Vogelstein illustrated decades ago a model of the adenoma-carcinoma sequence with gene alterations (3-5). Clinically, $\sim 65 \%$ of colorectal cancer patients survive for an average of 5 years $(1,2)$. However, when diagnosed with early stages and localized disease, the 5-year survival rate can reach $90.3 \%$, while the survival rate may be reduced to only $12.5 \%$ when patients are diagnosed with advanced stages with tumor distant metastasis $(6,7)$. The most common metastatic sites include the liver and peritonea and $20 \%$ of patients have liver metastases at the first diagnosis of colorectal (8-10). Moreover, tumor metastasis accounts for the majority of cancer-related deaths, and only $40 \%$ of patients are implicated to receive curable surgery. Thus, it is urgent to search for and identify novel biomarkers for the early detection of colorectal cancer, particularly for detection of tumor metastasis and progression.

Glia maturation factor $\gamma$ (GMFG) is a $17-\mathrm{kDa}$ protein and the GMF family of proteins includes GMFB and GMFG (11-14). GMFG levels are detectable in sera of healthy individuals without obvious difference between women and men and are enriched in various human organs, such as the thymus, spleen and colon (15). Structurally, GMFG protein belongs to the ADF/cofilin family and can modulate actin cytoskeleton reorganization in microvascular endothelial, human airway smooth muscle and ovarian cancer cells $(16,17)$. 
Moreover, GMFG can affect Toll-like receptor 4 (TLR4) signaling in macrophages, regulate chemotaxis of neutrophil and $\mathrm{T}$ lymphocytes $(18,19)$, and affect the angiogenic sprouting in zebra fish (20). Taken together, GMFG could regulate cell mobility and angiogenesis and therefore, we speculated that GMFG could play a role in cancer metastasis. Indeed, Zuo et al (14) showed that GMFG expression was able to influence migration and invasion of epithelial ovarian cancer. In addition, alteration and reduction in epithelial cell adhesion ability enhance epithelial-mesenchymal transition (EMT), and contribute to tumor cell migration, invasion and metastasis. E-cadherin (E-Cad) has the ability to regulate cell adhesion, whereas vimentin is usually overexpressed in carcinoma cells (21-23) and the matrix metalloproteinase (MMPs) family is critical in the modulation of tumor cell invasion and metastasis, particularly MMP2 and MMP9 (24), since these MMPs are enzymes that can digest collagen I and IV and laminin in the extracellular matrix (25-27). A previous study demonstrated that aberrant MMP2 gene-phenotype was associated with colorectal cancer prognosis (28).

In the present study, we first assessed GMFG expression in colorectal cancer cells and tissue specimens, and then explored the role of GMFG in the regulation of colorectal cancer cell migration and invasion. We aimed to provide insightful information in order to emphasize that detection of GMFG expression is a valuable biomarker with which to diagnose colorectal cancer metastasis and predict tumor progression.

\section{Materials and methods}

Cancer cell lines and colorectal cancer tissues. Different human cancer cell lines LoVo, HCT-116, LS174T, SW-620, SW-480, U87, U251, KBV, A549, MDA-231, HeLa, CaSki, SiHa, and MG-63 were obtained from the Research Center of Clinical Medicine, Nanfang Hospital (Guangzhou, China) and were maintained in Dulbecco's modified Eagle's medium (DMEM) containing 10\% fetal bovine serum (FBS) (both from HyClone, Logan, UT, USA) in a humidified incubator with $5 \% \mathrm{CO}_{2}$ at $37^{\circ} \mathrm{C}$.

Colorectal cancer tissue specimens were obtained from 68 patients who received surgery for tumor resection in Nanfang Hospital between December 2011 and January 2013. These patients did not receive any treatments before surgery and all cases were histologically diagnosed and retrospectively reviewed by two pathologists in the present study. The present study was approved by the Human Ethics Committee of Nanfang Hospital. In the present study, we collected paired normal and colorectal cancer tissue specimens and clinicopathological data from each patient. Tissue samples were stored in liquid nitrogen until use according to our previous study (29). Among these patients, 24 patients had rectal cancer and 44 had colon cancer, while 40 were at early stages of disease without tumor metastasis while 28 had different degrees of tumor metastasis according to the standard of the National Comprehensive Cancer Network (NCCN). The clinicopathological characteristics of the patients are presented in Table I.

Protein extraction and western blotting. Different cancer cell lines were grown and then collected from cell culture plates after being washed with phosphate-buffered saline
(PBS) 3 times and lysed in a lysis buffer containing $50 \mathrm{mM}$ Tris (pH 7.4), $150 \mathrm{mM} \mathrm{NaCl}, 1 \%$ sodium deoxycholate, $0.1 \%$ sodium dodecyl sulfate and phosphorylase and metalloproteinase inhibitor (Beyotime, Inc., Beijing, China). Colorectal tumor tissues were grinded on ice and then lysed in the same lysis buffer. Both types of cell lysis were then centrifuged and the concentration of protein samples was measured using the BCA protein assay kit (Beyotime). The protein samples were then treated with $5 \mathrm{X}$ loading buffer and boiled at $100^{\circ} \mathrm{C}$ for $5 \mathrm{~min}$, separated using sodium dodecyl sulfate-polyacrylamide gel electrophoresis (SDS-PAGE) on 12\% SDS-PAGE gels and transferred onto polyvinylidene fluoride (PVDF) membranes (Millipore, Billerica, MA, USA). The membranes were then blocked in 5\% skim milk solution in PBS and then incubated with different primary anti-human antibodies at $4^{\circ} \mathrm{C}$ overnight and the secondary antibody and enhanced chemiluminescence (ECL) kit according to a standard protocol. The primary antibodies were anti-GAPDH $(1: 1,000)$, anti-GMFG $(1: 1,000)$ (both from ProteinTech Group, Wuhan, China), anti-MMP2 $(1: 1,000)$, anti-E-Cad $(1: 1,000)$ and anti-vimentin $(1: 1,000)$ (all from Abcam, Cambridge, MA, USA).

Immunohistochemistry. Paraffin-embedded tissue specimens were sectioned into $4-\mu \mathrm{m}$ thick sections and put onto 3-aminopropyltriethoxysilane (APES)-coated glass slides. For immunohistochemistry, the sections were deparaffinized in xylene and rehydrated in a series of ethanol solution and submerged in tap-water. The antigen retrieval was then performed using an antigen unmasking solution, which was preheated in a microwave for $5 \mathrm{~min}$ and the sections in the buffer were heated at highest energy levels for $8 \mathrm{~min}$ in a microwave and cooled down to room temperature. Potential endogenous peroxidase activity was blocked in $3 \%$ hydrogen peroxide for $20 \mathrm{~min}$ and the sections were incubated in 5\% casein in PBS for $1 \mathrm{~h}$ to block non-specific antibody binding. The sections were then incubated at $4^{\circ} \mathrm{C}$ overnight with a rabbit anti-GMFG antibody (ProteinTech) at a dilution of 1:100 and subjected to a post primary block using the polymer penetration enhancer (Boshide, Wuhan, China) for $30 \mathrm{~min}$. Sections were then washed with PBS 5 min each for 3 times and further incubated at room temperature for $30 \mathrm{~min}$ with an anti-rabbit IgG-Poly-HRP (Merck, Kenilworth, NJ, USA). The sections were visualized with 3,3'-diaminobenzidine (DAB) solution (Boshide) and briefly counterstained with hematoxylin solution.

The immunostained sections were reviewed and scored by two investigators under a light microscope for intensity and percentage of staining. Protein localization was evaluated and classified as nuclear, cytoplasmic and plasmalemma locations. The percentage of positively stained tumor cells was graded as $0-10 \%(1+), 11-50 \%(2++)$ and $>50 \%$ stained cells $(3+++)$, while the staining intensity was graded as 1 (weakly positive), 2 (positive) and 3 (strongly positive). Subsequently, an immunostaining index was calculated by multiplying the staining intensity with the percentage of positive cells to reach negative ( 0 ), weak (1+), medium (2++) and strong $(3+++)$ expression of GMFG (Table I).

Quantitative reverse transcription-polymerase chain reaction (RT-qPCR). Total RNA was isolated from cells using 
Table I. GMFG expression and its association with the clinicopathological data from the colorectal cancer patients.

\begin{tabular}{|c|c|c|c|c|c|c|c|}
\hline \multirow[b]{2}{*}{ Clinical parameters } & \multirow[b]{2}{*}{ Cases } & \multicolumn{4}{|c|}{ Level of GMFG expression } & \multirow{2}{*}{$\begin{array}{l}\text { Positive } \\
\text { rate }(\%)\end{array}$} & \multirow[b]{2}{*}{ P-value } \\
\hline & & - & + & ++ & +++ & & \\
\hline Age (years) & & & & & & & 0.270 \\
\hline$\geq 60$ & 39 & 10 & 7 & 16 & 6 & 74.4 & \\
\hline$<60$ & 29 & 11 & 5 & 8 & 5 & 62.1 & \\
\hline Gender & & & & & & & 0.850 \\
\hline Male & 41 & 13 & 6 & 15 & 7 & 68.3 & \\
\hline Female & 27 & 8 & 6 & 9 & 4 & 70.4 & \\
\hline Lymph node metastasis & & & & & & & $0.013^{\mathrm{a}}$ \\
\hline Yes & 28 & 4 & 7 & 12 & 5 & 85.7 & \\
\hline No & 40 & 17 & 5 & 12 & 6 & 57.5 & \\
\hline Tumor differentiation & & & & & & & 0.480 \\
\hline Well & 20 & 8 & 4 & 5 & 3 & 60.0 & \\
\hline Moderate & 42 & 12 & 7 & 17 & 6 & 71.4 & \\
\hline Poor & 6 & 1 & 1 & 2 & 2 & 83.3 & \\
\hline Total no. & & & & & & & $0.046^{\mathrm{a}}$ \\
\hline Normal & 68 & 40 & 13 & 8 & 7 & 41.2 & \\
\hline Cancer & 68 & 21 & 12 & 24 & 11 & 69.1 & \\
\hline
\end{tabular}

${ }^{\mathrm{a}} \mathrm{P}<0.05$. GMFG, glia maturation factor $\gamma$.

TRIzol reagent (Invitrogen, Carlsbad, CA, USA) and reversely transcribed into cDNA using 5X PrimeScript RT Master Mix (Takara, Dalian, China) according to the manufacturer's protocols. After that, these cDNA samples were subjected to qPCR amplification using 2X SYBR Premix Ex Taq (Takara) in the 7500 ABI Real-Time PCR System (Applied Biosystems, Foster City, CA, USA). The amplification conditions were $95^{\circ} \mathrm{C}$ for $30 \mathrm{sec}, 95^{\circ} \mathrm{C}$ for $5 \mathrm{sec}$, and $60^{\circ} \mathrm{C}$ for $34 \mathrm{sec}$ for 40 cycles and the relative level of mRNA expression was calculated using the $2^{-\Delta \Delta \mathrm{Ct}}$ method after normalization to the level of GAPDH mRNA. The primers used were: GMFG, 5'-CGC GGG AAG TAA AAA CAGG C-3' and 5'-GGT CTC GTT GAG GTC GTC TG-3'; and GAPDH, 5'-AGA AGG TGG GGC TCA TTT G-3 and 5-AGG GGC CAT CCA CAGT CTT C-3'.

GMFG RNA interference. To knockdown GMFG expression in colorectal cancer cells, we used GMFG siRNA purchased from Ruibo Co. (Guangzhou, China) and transfected GMFG siRNA or negative control (NC) siRNA into the colorectal cells for $24 \mathrm{~h}$ using Hylimax (Dojindo, Kumamoto, Japan) in Opti-MEM (Thermo Fisher Scientific, Waltham, MA, USA) according to the manufacturer's instructions and assayed. The NC siRNA was random siRNA duplex sequences.

Tumor cell migration and invasion assays. For the migration assay, $5 \times 10^{4}$ cells in a serum-free medium were seeded onto an uncoated filter with $8.0-\mu \mathrm{m}$ pores (Corning, Corning, NY, USA), whereas $1 \times 10^{5}$ cells were seeded onto a filter with $8.0 \mathrm{~mm}$ pores precoated the extracellular matrix (BD Biosciences, Franklin Lakes, NJ, USA) for the invasion assays. The bottom chambers were filled with $10 \%$ FBS and cultured for $24 \mathrm{~h}$. In separated experiments, different concentrations of recombinant GMFG $(0,5,10,20$ and $30 \mu \mathrm{g} / \mathrm{ml})$ were added into the bottom chambers. At the end of the experiments, cells on the surface of the filters were removed using cotton swabs and PBS, while cells that had migrated or invaded into the low surface of the filters were fixed and stained with crystal violet (Beyotime). The numbers of migrated and invaded cells were counted under a microscope for 10 of $20 \mathrm{x}$ microscopic fields and averaged.

ELISA detection of GMFG in cell culture medium. The level of GMFG in cell culture medium was measured using the GMFG ELISA kit (Cusabio Biotech Co., Ltd., Wuhan, China) according to the manufacturer's protocol. GMFG levels were expressed as mean \pm SD of triplicate experiments and repeated at least 3 times.

Statistical analysis. Statistical analyses were performed using the SPSS 13.0 statistical software (SPSS, Chicago, IL, USA). The data are expressed as mean \pm SD and were statistically analyzed using one-way ANOVA and $\chi^{2}$ test. $\mathrm{P}<0.05$ indicates statistically significant results.

\section{Results}

Expression of GMFG in different cancer cell lines and colorectal cancer tissue specimens. In the present study, we first detected GMFG expression in 14 different cancer cell lines and found that GMFG was expressed in the majority of the cell lines (Fig. 1A). GMFG protein was preferentially expressed in U87, U251, KBV, MDA-231, SiHa and LoVo cells, 
A

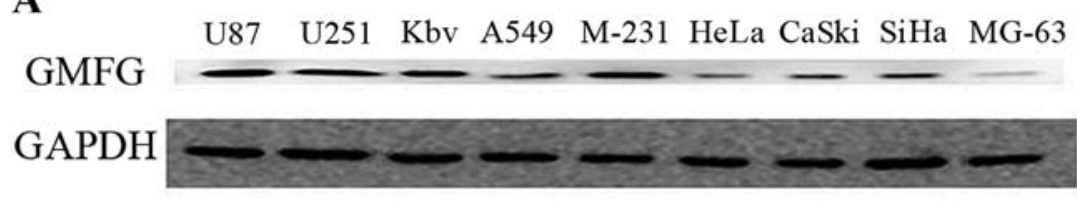

B

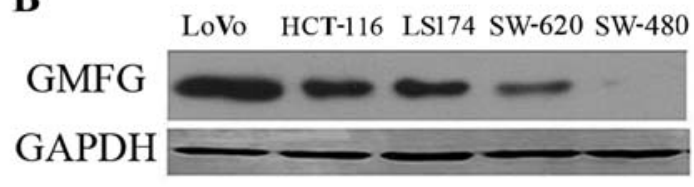

Figure 1. Expression of GMFG protein in various types of human cancer cell lines. (A) Western blot analysis of GMFG expression in 9 different cancer cell lines. (B) Western blot analysis of GMFG expression in 5 different colorectal cell lines.

$\mathbf{A}$

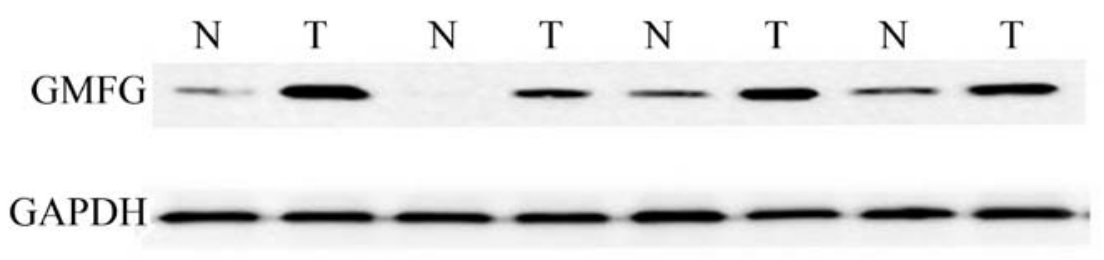

B
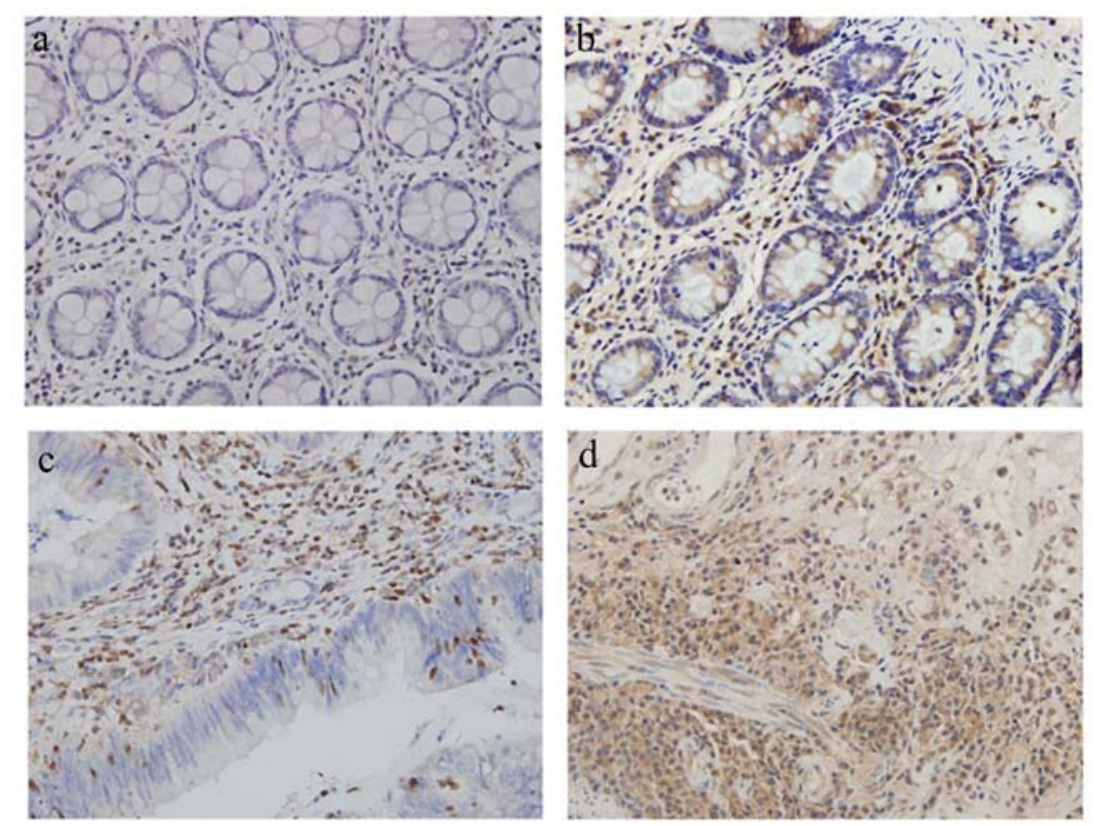

Figure 2. Expression of GMFG in colorectal cancer and normal tissues. (A) Western blot analysis of GMFG expression in paired normal colorectal (N) and colorectal cancer tissues (T) from 68 patients (data for 4 paired samples are shown). (B) Immunohistochemistry. The data showed differential expression of GMFG in colorectal adenocarcinoma and normal adjacent tissues (a, normal tissue; b, well-differentiated colorectal cancer tissue; c moderately differentiated colorectal cancer tissues; and d, poorly differentiated colorectal cancer tissue). Original magnification, x100.

while colorectal cancer cell line LoVo expressed the highest level of GMFG compared with the other cell lines (Fig. 1B).

We then analyzed GMFG expression in 68 colorectal cancer surgical samples and found that 42 samples $(61.8 \%)$ expressed a high level of GMFG protein using western blotting compared with the adjacent non-tumor tissues, while 14 samples $(20.6 \%)$ showed no obvious difference, whereas 12 samples (17.6\%) had lower levels of GMFG compared with normal tissues (Fig. 2A).
Immunohistochemical data showed that GMFG was highly expressed in colorectal tissues compared to normal tissues (Fig. 2B). GMFG protein was mainly localized in mesenchymal cells of the non-tumor colorectal tissues and in the gland cells in the tumor tissues. We then associated GMFG expression with clinicopathological parameters from these patients and found that GMFG was associated with lymph node metastasis ( 85.7 vs. $57.5 \%$ in non-metastatic cases; Table I), but there was no statistically significant difference in 
A

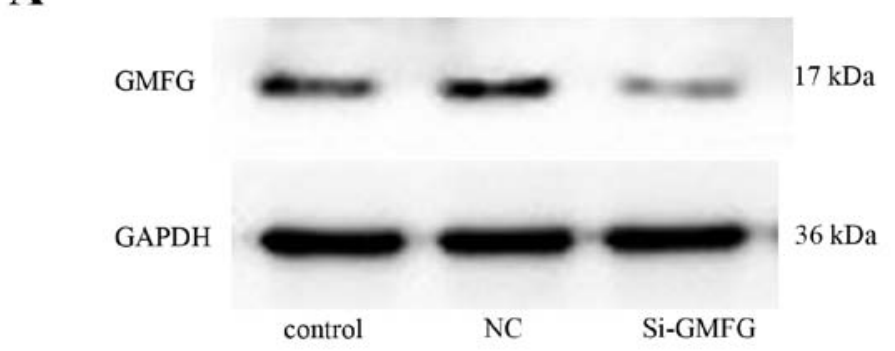

C

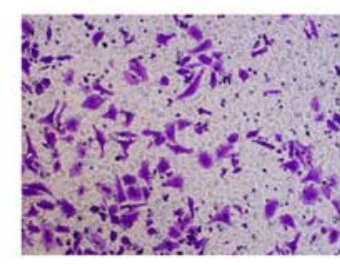

control

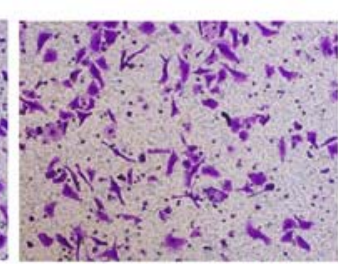

$\mathrm{NC}$

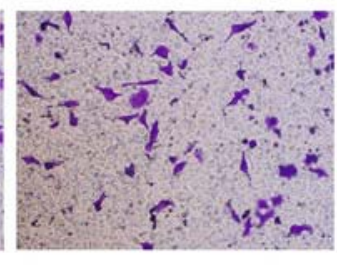

Si-GMFG

B
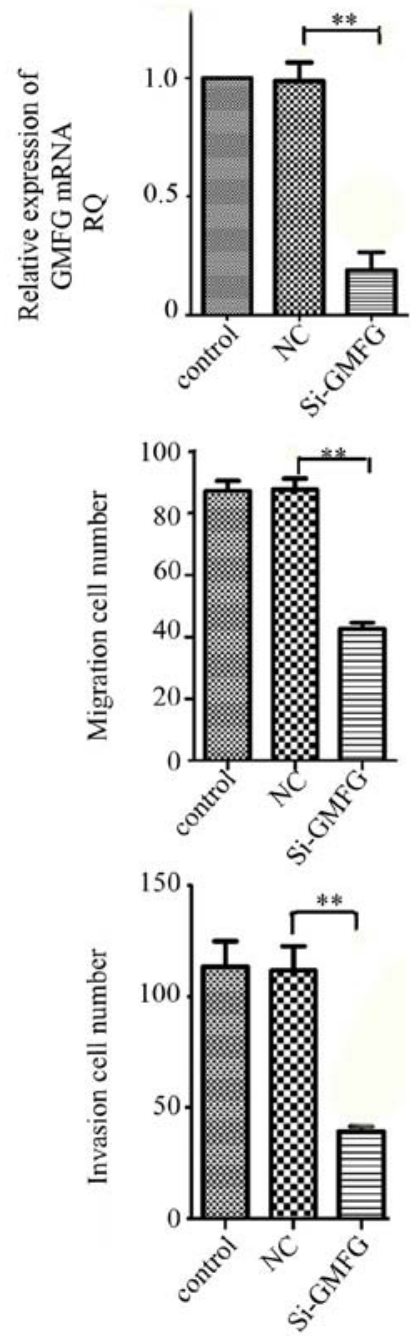

Figure 3. Effects of GMFG knockdown on the regulation of colorectal LoVo cell migration and invasion. (A) Western blot analysis of GMFG expression in LoVo cells after transfection with GMFG siRNA vs. negative control siRNA. (B) RT-qPCR analysis of the GMFG mRNA level in LoVo cells after transfection with GMFG siRNA vs. negative control siRNA. (C) Tumor cell migration assay. LoVo cells were grown and transiently transfected with GMFG siRNA vs. negative control siRNA, and then subjected to Transwell migration assay. The graph shows the summarized data. (D) Tumor cell invasion assay. LoVo cells were grown and transiently transfected with GMFG siRNA vs. negative control siRNA and then subjected to Transwell migration assay. The graph shows summarized data of the means $\pm \mathrm{SD}$ of $\mathrm{n}=3$ experiments; ${ }^{* *} \mathrm{P}<0.01$ vs. the control cells.

GMFG expression associated with patient gender and age and tumor differentiation (Table I).

Suppression of LoVo cell migration and invasion abilities after knockdown of GMFG expression. We then assessed the effects of GMFG knockdown in colorectal cancer cells using GMFG siRNA. Our data showed that GMFG siRNA significantly silenced GMFG expression in the LoVo cells (Fig. 3A and B). We then performed the Transwell assay to assay the altered ability of tumor cell migration and invasion, and found that both tumor cell migration and invasion capacities were reduced after knockdown of GMFG expression (Fig. 3C and D).

Induction of LoVo cell migration and invasion by exogenous GMFG treatment. We further determined whether addition of exogenous GMFG could affect LoVo cell migration. As shown in Fig. 4A, the number of LoVo cells was increased after addition of exogenous GMFG and the maximal GMFG concentration was $20 \mu \mathrm{g} / \mathrm{ml}$, although the level of LoVo cell migration did not obviously increase as GMFG concentration continued to rise.

Induction of LoVo cell migration after co-culture with HUVECs. As discussed in the Introduction section, GMFG can promote angiogenesis. We, thus, co-cultured LoVo cells with human umbilical vein endothelial cells (HUVECs). Our data showed that the migration capacity of the LoVo cells was increased after being co-cultured with the HUVECs (Fig. 4B). However, addition of the GMFG antibody into this co-culture downregulated LoVo cell migration (Fig. 4C). Moreover, knockdown of GMFG expression in HUVECs and then co-culture with LoVo cells showed a decrease in LoVo cell migration compared with the NC group (Fig. 4D). However, we failed to detect GMFG levels in cell culture medium in our cancer cell lines by using GMFG ELISA kit (data not shown).

Inhibition of MMP2 expression and reversal of EMT after knockdown of GMFG expression in LoVo cells. Western blot 
A a b c d e

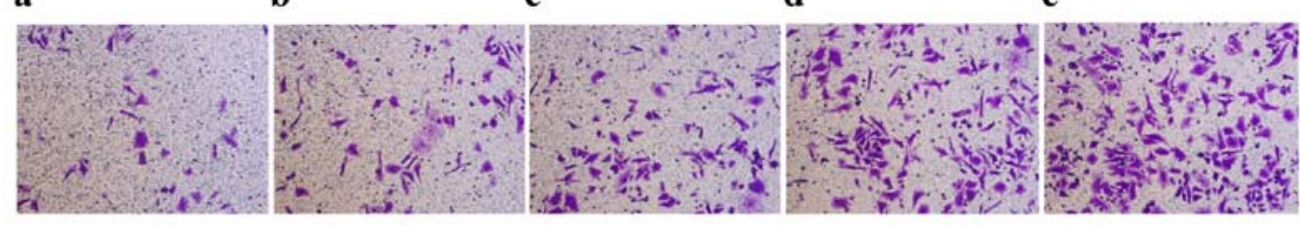

B

C

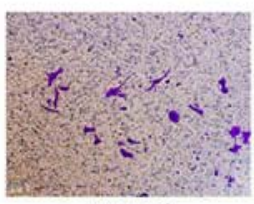

control

D

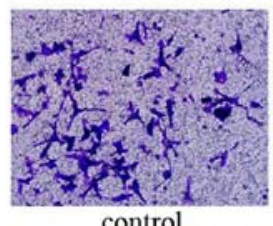

control

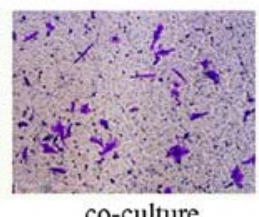

co-culture

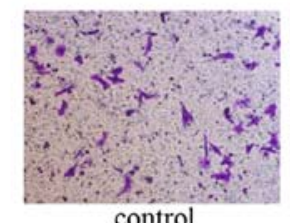

control

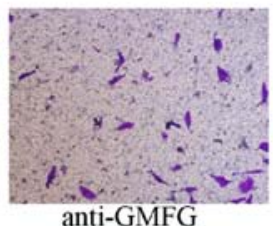

anti-GMFG
$\mathbf{E}$

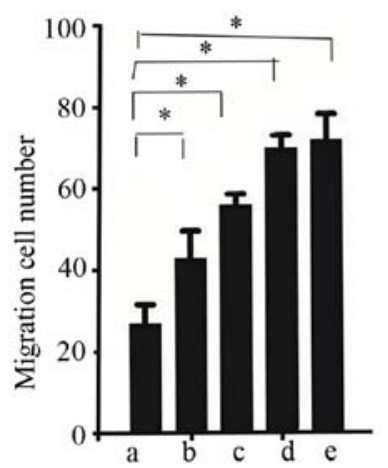

F

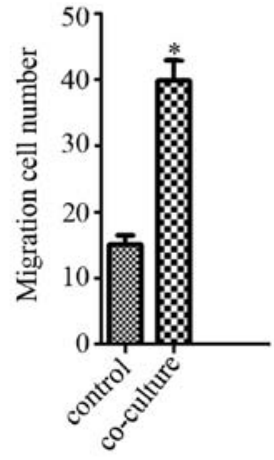

G

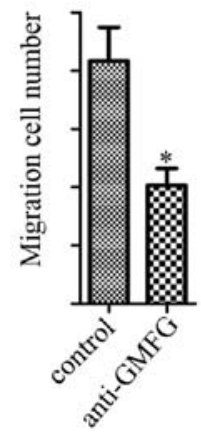

H

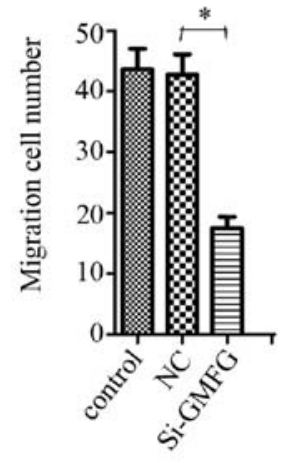

Figure 4. Effects of different GMFG modulation on the regulation of LoVo cell migration as detected by Transwell migration assay. (A) LoVo cells were grown and then treated with different concentrations of exogenous $\mathrm{GMFG}$ and then subjected to Transwell migration assay. a, $0 \mu \mathrm{g} / \mathrm{ml} ; \mathrm{b}, 5 \mu \mathrm{g} / \mathrm{ml} ; \mathrm{c}, 10 \mu \mathrm{g} / \mathrm{ml}$; $\mathrm{d}, 20 \mu \mathrm{g} / \mathrm{ml}$; and e, $30 \mu \mathrm{g} / \mathrm{ml}$. (B) LoVo cells were co-cultured with HUVECs and then subjected to Transwell migration assay. (C) LoVo cells were grown and then treated with the anti-GMFG antibody (10 $\mu \mathrm{l} /$ well) and then subjected to Transwell migration assay. (D) LoVo cells were co-cultured with HUVECs that had first been transfected for $24 \mathrm{~h}$ with GMFG siRNA or negative control siRNA for $24 \mathrm{~h}$ and then subjected to Transwell migration assay. (E-H) Quantitative data of A-D, respectively. All data are expressed as means \pm SD of $n=3$ experiments; ${ }^{*} \mathrm{P}<0.05$ vs. the control cells.

analysis revealed that knockdown of GMFG expression downregulated expression of E-Cad and MMP2, but upregulated the vimentin level in the LoVo cells (Fig. 5), indicating that GMFG promoted tumor cell EMT while silencing of GMFG reversed the EMT phenotype in the LoVo cells.

\section{Discussion}

In the present study, we first assessed GMFG expression in colorectal cancer cell lines and tissue specimens, and then explored the role of GMFG in the regulation of colorectal cancer cell migration and invasion in vitro. We found that GMFG protein was expressed in 14 different common human cancer cell lines and the highest GMFG level was noted in colorectal cancer LoVo cell line. Furthermore, we also found that GMFG was highly expressed in colorectal cancer tissue samples, particularly in patients with lymph node metastasis (85.7 vs. $57.5 \%$ of non-metastatic patients). However, knockdown of GMFG expression or anti-GMFG antibody reduced the migration and invasion abilities of the LoVo cells, whereas GMFG treatment induced LoVo cell migration capacity, which is consistent with a recent study reported by Zuo et al who demonstrated that GMFG expression promoted migration and invasion of epithelial ovarian cancer cells (14). In addition, our current data also showed that knockdown of GMFG expression altered expression of tumor EMT-related proteins, such as MMP2, E-cadherin and vimentin. Since GMFG protein functions to reorganize the actin cytoskeleton and previous 

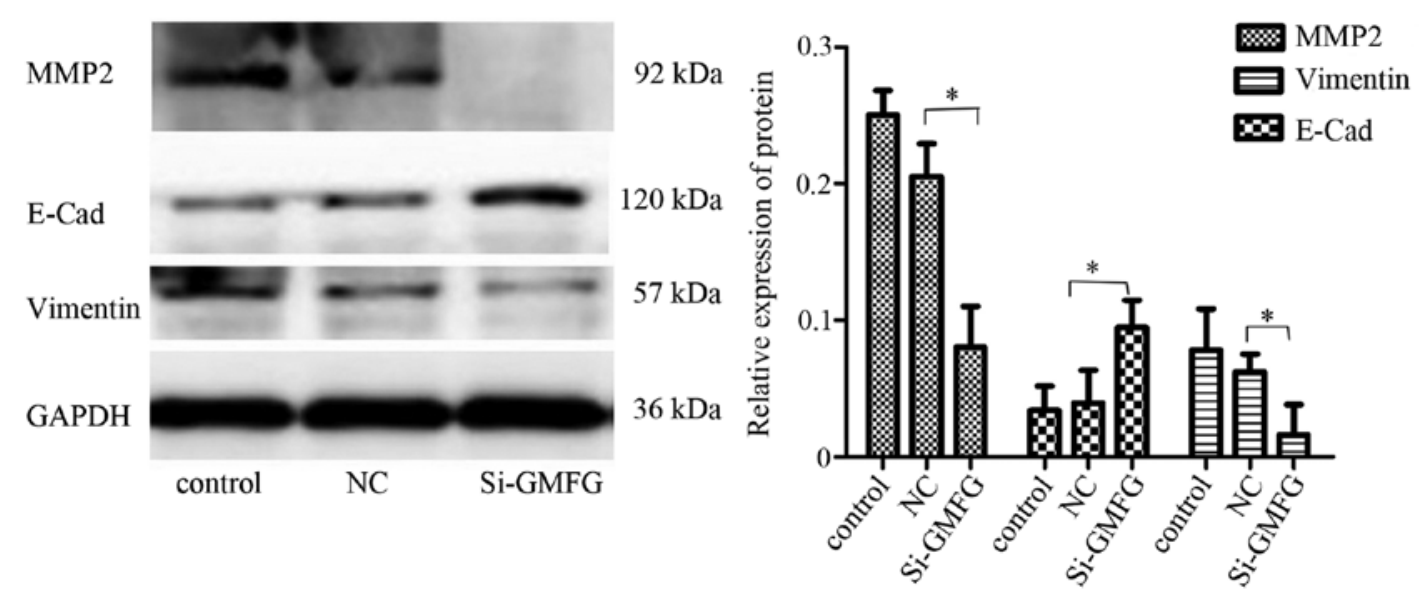

Figure 5. Effect of GMFG siRNA on expression of MMP2, E-cadherin (E-Cad) and vimentin in LoVo cells. LoVo cells were grown and then transiently transfected with GMFG siRNA or negative control siRNA and then subjected to western blotting. The graph shows the summarized data of the western blot analyses; ${ }^{\mathrm{P}}<0.05$.

studies have shown that GMFG induced the migration of human T lymphocytes $(16,19)$, our current data suggest that GMFG-induced colorectal cancer cell migration and invasion could be through GMFG-altered tumor cell EMT, although future study is needed to confirm this.

Furthermore, the LoVo cell line was derived from a patient with metastatic colon cancer and has a high ability to invade and metastasize, while the SW480 cell line was established from a patient with primary colon adenocarcinoma. The present study showed that expression of GMFG protein was higher in the LoVo cells than that in the SW480 cell line. In tissue samples, we also found a similar phenomenon, i.e., GMFG protein was higher in tissues with metastatic colorectal cancer. These results suggest that detection of GMFG protein could be used as a biomarker for detection of colorectal cancer, particularly, metastatic colorectal cancer.

Previous studies have confirmed that EMT is an important early step in the conversion of tumor cells into a migratory population capable of systemic tumor metastasis (30-32). At the molecular level, EMT is associated with a gain in mesenchymal markers, including vimentin and $\mathrm{N}$-cadherin but a loss of adherent junction proteins, such as E-cadherin (33-36). In the present study, we found that knockdown of GMFG expression upregulated E-cadherin expression but downregulated vimentin and MMP2 expression in LoVo cells. To the best of our knowledge, MMP2 is a key protein in the process of tumor cell migration and invasion (37-39). Our current finding provides a more plausible explanation for highly expressed GMFG protein in most colorectal cancer tissues. However, it is true that we do not know the mechanism of how GMFG mediated alteration of the expression of these proteins. This mechanism could involve GMFG activation of cell receptors or modulation of integrin expression or functions. Regardless, the targeting of GMFG expression or function may be a novel strategy by which to control colorectal cancer progression.

In addition, the present study for the first time confirmed that exogenous GMFG treatment promoted colorectal cancer cell migration and that co-culture of LoVo cells with HUVECs also promoted tumor cell migration, whereas knockdown of GMFG expression or anti-GMFG antibody decreased colorectal cancer migration. We then assessed whether HUVECs could secrete GMFG into the cell culture medium to play its role in the promotion of colorectal cancer cell migration. However, we could not detect expression of GMFG in the culture media, although we cannot exclude the role of secreted GMFG in the regulation of colorectal cancer migration and invasion, which may be due to the fact that ELISA was unable to detect the low GMFG level in the growth media. Thus, we will continue to investigate the underlying molecular mechanism by which GMFG modulates colorectal cancer invasion and metastasis.

In conclusion, the present study demonstrated that GMFG expression is associated with colorectal cancer metastasis to lymph nodes ex vivo and knockdown of GMFG expression or the anti-GMFG antibody could reduce tumor cell migration and invasion in vitro. Thus, GMFG should be further evaluated as a diagnostic marker in the early detection of colorectal cancer metastasis. The targeting of GMFG expression may suppress colorectal cancer progression.

\section{References}

1. Siegel RL, Miller KD and Jemal A: Cancer statistics, 2016. CA Cancer J Clin 66: 7-30, 2016.

2. Ferlay J, Steliarova-Foucher E, Lortet-Tieulent J, Rosso S, Coebergh JW, Comber H, Forman D and Bray F: Cancer incidence and mortality patterns in Europe: Estimates for 40 countries in 2012. Eur J Cancer 49: 1374-1403, 2013.

3. Shih IM, Zhou W, Goodman SN, Lengauer C, Kinzler KW and Vogelstein B: Evidence that genetic instability occurs at an early stage of colorectal tumorigenesis. Cancer Res 61: 818-822, 2001.

4. Höglund M, Gisselsson D, Hansen GB, Säll T, Mitelman F and Nilbert M: Dissecting karyotypic patterns in colorectal tumors: Two distinct but overlapping pathways in the adenoma-carcinoma transition. Cancer Res 62: 5939-5946, 2002.

5. Hagland HR, Berg M, Jolma IW, Carlsen A and Søreide K: Molecular pathways and cellular metabolism in colorectal cancer. Dig Surg 30: 12-25, 2013.

6. Howlader N, Noone AM, Krapcho M, Garshell J, Neyman N, Altekruse SF, Kosary CL, Yu M, Ruhl J, Tatalovich Z, et al (eds): SEER Cancer Statistics Review, 1975-2010. National Cancer Institute, Bethesda, MD, 2013. http://seer.cancer.gov/ csr/1975_2010/.

7. DeBerardinis RJ, Lum JJ, Hatzivassiliou G and Thompson CB: The biology of cancer: Metabolic reprogramming fuels cell growth and proliferation. Cell Metab 7: 11-20, 2008. 
8. Willett WC: Diet and cancer: An evolving picture. JAMA 293: 233-234, 2005.

9. de Jong AE, Morreau H, Nagengast FM, Mathus-Vliegen EM, Kleibeuker JH, Griffioen G, Cats A and Vasen HF: Prevalence of adenomas among young individuals at average risk for colorectal cancer. Am J Gastroenterol 100: 139-143, 2005.

10. Haggar FA and Boushey RP: Colorectal cancer epidemiology: Incidence, mortality, survival, and risk factors. Clin Colon Rectal Surg 22: 191-197, 2009.

11. Mao M, Fu G, Wu JS, Zhang QH, Zhou J, Kan LX, Huang QH, He KL, Gu BW, Han ZG, et al: Identification of genes expressed in human $\mathrm{CD} 34^{+}$hematopoietic stem/progenitor cells by expressed sequence tags and efficient full-length cDNA cloning. Proc Natl Acad Sci USA 95: 8175-8180, 1998.

12. Asai K, Fujita K, Yamamoto M, Hotta T, Morikawa M, Kokubo M, Moriyama A and Kato T: Isolation of novel human cDNA (hGMF-gamma) homologous to Glia Maturation Factorbeta gene. Biochim Biophys Acta 1396: 242-244, 1998.

13. Shi Y, Chen L, Liotta LA, Wan HH and Rodgers GP: Glia maturation factor gamma (GMFG): A cytokine-responsive protein during hematopoietic lineage development and its functional genomics analysis. Genomics Proteomics Bioinformatics 4 145-155, 2006.

14. Zuo P, Ma Y, Huang Y, Ye F, Wang P, Wang X, Zhou C, Lu W, Kong B and Xie X: High GMFG expression correlates with poor prognosis and promotes cell migration and invasion in epithelial ovarian cancer. Gynecol Oncol 132: 745-751, 2014.

15. Inagaki M, Aoyama M, Sobue K, Yamamoto N, Morishima $T$, Moriyama A, Katsuya $\mathrm{H}$ and Asai K: Sensitive immunoassays for human and rat GMFB and GMFG, tissue distribution and age-related changes. Biochim Biophys Acta 1670: 208-216, 2004.

16. Ikeda K, Kundu RK, Ikeda S, Kobara M, Matsubara H and Quertermous T: Glia maturation factor-gamma is preferentially expressed in microvascular endothelial and inflammatory cells and modulates actin cytoskeleton reorganization. Circ Res 99: 424-433, 2006.

17. Wang T, Cleary RA, Wang R and Tang DD: Glia maturation factor- $\gamma$ phosphorylation at Tyr-104 regulates actin dynamics and contraction in human airway smooth muscle. Am J Respir Cell Mol Biol 51: 652-659, 2014.

18. Aerbajinai W, Liu L, Chin K, Zhu J, Parent CA and Rodgers GP: Glia maturation factor- $\gamma$ mediates neutrophil chemotaxis. J Leukoc Biol 90: 529-538, 2011.

19. Lippert DN and Wilkins JA: Glia maturation factor gamma regulates the migration and adherence of human $\mathrm{T}$ lymphocytes. BMC Immunol 13: 21, 2012.

20. Zuo P, Fu Z, Tao T, Ye F, Chen L, Wang X, Lu W and Xie X: The expression of glia maturation factors and the effect of glia maturation factor- $\gamma$ on angiogenic sprouting in zebrafish. Exp Cell Res 319: 707-717, 2013.

21. Satelli A and Li S: Vimentin in cancer and its potential as a molecular target for cancer therapy. Cell Mol Life Sci 68: 3033-3046, 2011.

22. Arias AM: Epithelial mesenchymal interactions in cancer and development. Cell 105: 425-431, 2001.
23. Bates RC and Mercurio AM: The epithelial-mesenchymal transition (EMT) and colorectal cancer progression. Cancer Biol Ther 4: 365-370, 2005.

24. Turpeenniemi-Hujanen T: Gelatinases (MMP-2 and -9) and their natural inhibitors as prognostic indicators in solid cancers. Biochimie 87: 287-297, 2005.

25. Shi M, Yu B, Gao H, Mu J and Ji C: Matrix metalloproteinase 2 overexpression and prognosis in colorectal cancer: A metaanalysis. Mol Biol Rep 40: 617-623, 2013.

26. Mook OR, Frederiks WM and Van Noorden CJ: The role of gelatinases in colorectal cancer progression and metastasis. Biochim Biophys Acta 1705: 69-89, 2004.

27. Théret N, Lehti K, Musso O and Clément B: MMP2 activation by collagen I and concanavalin A in cultured human hepatic stellate cells. Hepatolog y 30: 462-468, 1999.

28. Langers AM, Sier CF, Hawinkels LJ, Kubben FJ, van Duijn W, van der Reijden JJ, Lamers CB, Hommes DW and Verspaget HW: MMP-2 geno-phenotype is prognostic for colorectal cancer survival, whereas MMP-9 is not. Br J Cancer 98: 1820-1823, 2008.

29. Wang Z, Wang L, Hu J, Fan R, Zhou J, Wang L and Zhong J: RARRES3 suppressed metastasis through suppression of MTDH to regulate epithelial-mesenchymal transition in colorectal cancer. Am J Cancer Res 5: 1988-1999, 2015.

30. Thiery JP: Epithelial-mesenchymal transitions in tumour progression. Nat Rev Cancer 2: 442-454, 2002.

31. Kalluri R and Weinberg RA: The basics of epithelial-mesenchymal transition. J Clin Invest 119: 1420-1428, 2009.

32. Bravo-Cordero JJ, Hodgson L and Condeelis J: Directed cell invasion and migration during metastasis. Curr Opin Cell Biol 24: 277-283, 2012.

33. Grünert $S$, Jechlinger $M$ and Beug H: Diverse cellular and molecular mechanisms contribute to epithelial plasticity and metastasis. Nat Rev Mol Cell Biol 4: 657-665, 2003.

34. Pohl M, Radacz Y, Pawlik N, Schoeneck A, Baldus SE, Munding J, Schmiegel W, Schwarte-Waldhoff I and Reinacher-Schick A: SMAD4 mediates mesenchymal-epithelial reversion in SW480 colon carcinoma cells. Anticancer Res 30: 2603-2613, 2010.

35. Micalizzi DS and Ford HL: Epithelial-mesenchymal transition in development and cancer. Future Oncol 5: 1129-1143, 2009.

36. Huber MA, Kraut $\mathrm{N}$ and Beug $\mathrm{H}$ : Molecular requirements for epithelial-mesenchymal transition during tumor progression. Curr Opin Cell Biol 17: 548-558, 2005.

37. Bae GY, Choi SJ, Lee JS, Jo J, Lee J, Kim J and Cha HJ: Loss of E-cadherin activates EGFR-MEK/ERK signaling, which promotes invasion via the ZEB1/MMP2 axis in non-small cell lung cancer. Oncotarget 4: 2512-2522, 2013.

38. Onder TT, Gupta PB, Mani SA, Yang J, Lander ES and Weinberg RA: Loss of E-cadherin promotes metastasis via multiple downstream transcriptional pathways. Cancer Res 68: 3645-3654, 2008.

39. Lu L, Xue X, Lan J, Gao Y, Xiong Z, Zhang H, Jiang W, Song W and Zhi Q: MicroRNA-29a upregulates MMP2 in oral squamous cell carcinoma to promote cancer invasion and anti-apoptosis. Biomed Pharmacother 68: 13-19, 2014. 